\section{Morbidade hospitalar em crianças indígenas Suruí menores de dez anos, Rondônia, Brasil: 2000 a 2004}

\section{Hospital morbidity in Suruí indigenous children under ten years old, Rondonia, Brazil, 2000 to 2004}

Jesem Douglas Yamall Orellana 1

Paulo Cesar Basta 2

Ricardo Ventura Santos 3

Carlos E.A. Coimbra Jr. 4

\section{Abstract}

Objectives: to analyze the pattern of hospital morbidity of Suruí Indian children at the local network of the Brazilian Universal Health System.

Methods: inpatient data were obtained for 380 children <10 years old hospitalized during 2000 to 2004 at the two hospitals in the city of Cacoal, State of Rondonnia, to which Indian patients are referred to Hospital Materno-Infantil (public) and Hospital Infantil e Maternidade Menino Jesus (private). Hospitalization causes were classified according to the International Disease Classification 10th revision.

Results: diseases of the respiratory system - Chapter $X(58.2 \%)$ were the most frequent cause of hospitalization, followed by certain infectious and parasitic diseases - Chapter I (35.0\%) and other conditions developed in the perinatal period - Chapter XVI (3.2\%). All other causes contributed with less that $1.5 \%$ of the total number of admissions, totaling less than $4.0 \%$ combined. The majority of hospital admissions (65.5\%) were observed in children $<2$ years old. The average number of hospitalization days was significantly higher in public hospital.

Conclusions: the hospital morbidity pattern observed in Surui children not only discloses their precarious health conditions, but it also points to structural deficiencies at the primary healthcare level. The authors understand that more research is needed to better grasp the epidemiologic background associated with hospitalization causes in Indigenous populations. This information is of paramount value in order to better plan the health interventions aimed at these populations. Key words Hospitalization, Morbidity, Health services, Child health, Indians South American

\section{Resumo}

Objetivos: analisar o perfil de morbidade hospitalar de crianças indígenas atendidas na rede de serviços do Sistema Único de Saúde.

Métodos: foram investigados 380 registros de internação hospitalar de crianças indígenas Suruí menores de 10 anos de idade atendidas na rede hospitalar do município de Cacoal, Rondônia, a partir de dados coletados no Hospital Materno-Infantil, instituição pública, e no Hospital Infantil e Maternidade Menino Jesus, instituição particular, no período de 2000 a 2004. Esses dois hospitais concentram o atendimento a indígenas no município. Utilizou-se a Classificação Internacional de Doenças (CID), 10. a revisão, para a classificação dos diagnósticos.

Resultados: as doenças do aparelho respiratório Cap. X $(58,2 \%)$ figuraram como o principal motivo de internação hospitalar. Doenças infecciosas e parasitárias (Cap I) apresentaram-se como a segunda maior causa $(35,0 \%)$ e afeç̧ões originadas no período perinatal (Cap XVI) como a terceira $(3,2 \%)$. Outras causas contribuíram individualmente com menos de $1,5 \%$ do total, que somadas não atingiram 4\%. A maioria das internações $(65,5 \%)$ foram de crianças $<2$ anos de idade. O tempo médio de internação foi significativamente superior no hospital público em relação ao privado.

Conclusões: o perfil de morbidade hospitalar observado nas crianças Suruí evidencia não somente a precariedade das suas condições de saúde, como também problemas estruturais no que tange às ações de atenção básica. Recomenda-se a realização de estudos que visem aprofundar aspectos epidemiológicos relacionados às internações hospitalares em populações indígenas, fundamentais para o desenvolvimento de ações de saúde mais adequadas. Palavras-chave Hospitalização, Morbidade, Serviços de Saúde, Saúde da Criança, Índios Sul-Americanos 


\section{Introdução}

As estimativas sobre o tamanho da população indígena no Brasil, a depender da fonte, variam entre 350 a 700 mil indivíduos, sinalizando para uma expressiva expansão demográfica nas últimas décadas, em larga medida associada a elevadas taxas de fecundidade. 1 Vale destacar que aproximadamente 40 a $50 \%$ desse contingente populacional é composto por menores de 15 anos de idade.

Recentes investigações apontam para um quadro de extrema fragilidade das condições de saúde e nutrição dos povos indígenas no Brasil, principalmente das crianças, quando comparadas às não-indígenas.2,3 Dados do Censo Demográfico de 2000 indicam que, para os auto-declarados indígenas nas áreas urbanas e rurais do país, a taxa de mortalidade infantil foi de 51,4 por mil, valor bem acima da média nacional $\left(30,1\right.$ por mil). ${ }^{4}$ Estudos realizados com comunidades indígenas específicas também demonstram diferenciais importantes entre a mortalidade infantil indígena e não-indígena. ${ }^{3}$

Devido a deficiências nas bases e nos sistemas de informação em saúde, incluindo o Sistema de Informação da Atenção à Saúde Indígena (SIASI), 3,5 alguns autores têm buscado, indiretamente, gerar conhecimentos sobre os perfis de morbidade por meio da análise de registros de internações hospitalares.6-8 Essas pesquisas têm identificado como principais causas de internação de crianças indígenas as doenças do aparelho respiratório e as infecciosas e parasitárias (especialmente as diarréias), além de verificarem que crianças menores de cinco anos, proporcionalmente, são mais internadas que outros grupos etários.

O objetivo deste estudo foi analisar o perfil de morbidade hospitalar das crianças indígenas Suruí, Rondônia, atendidas na rede de serviços do Sistema Único de Saúde (SUS) no período de 2000 a 2004, por ocasião de pesquisa sobre a epidemiologia da tuberculose $\mathrm{e}^{9}$ e da desnutrição ${ }^{10}$ realizada na comunidade.

\section{Métodos}

O povo indígena Suruí encontra-se distribuído em 11 aldeias situadas na Terra Indígena Sete de Setembro $\left( \pm 60^{\circ}-61^{\circ} \mathrm{W} ; 10^{\circ}-12^{\circ} \mathrm{S}\right)$, na divisa entre Mato Grosso e Rondônia, Brasil. Em fevereiro-março de 2005 , por ocasião da pesquisa de campo, a população totalizava 993 pessoas, das quais $348(35,0 \%)$ eram crianças menores de 10 anos.

Quanto à inserção no subsistema de atenção à saúde indígena, os Suruí estão jurisdicionados ao pólo-base Cacoal, em Rondônia que integra o Distrito Sanitário Especial Indígena (DSEI) Vilhena. Aos pólos-base compete coordenar as equipes multidisciplinares de saúde (EMS), responsáveis pelas ações de saúde nas aldeias. À época do estudo, o pólo-base Cacoal não contava com médicos em sua EMS e as intervenções caracterizavam-se como pontuais e sem continuidade. Os casos de maior complexidade são encaminhados à Casa de Saúde do Índio, em Cacoal, onde são realizados pequenos procedimentos de enfermagem e, quando necessário, encaminhamentos à rede SUS.

Os dados analisados neste trabalho foram obtidos em dois hospitais da rede SUS, situados em Cacoal. Ambos os hospitais são de pequeno porte e se caracterizam por atendimento especializado de média complexidade. No Hospital Materno-Infantil (HMI), único hospital público da cidade, os dados foram colhidos diretamente das Autorizações de Internação Hospitalar (AIH), disponíveis no Serviço de Estatística Médica da unidade. No Hospital Infantil e Maternidade Menino Jesus (HIMMJ), instituição privada, conveniada ao SUS, os dados, para o período de 2000 a 2004, foram obtidos de um livro-resumo de internações hospitalares exclusivo para crianças, devido a não disponibilização das AIH para consulta. Esses dois hospitais concentram o atendimento a indígenas no município.

A partir dos registros hospitalares foram colhidas as seguintes variáveis: nome, data de internação, data de alta, diagnóstico principal, idade, sexo e aldeia de procedência. Em caso de dúvidas, procedeu-se a checagem dos dados nos prontuários e no Sistema de Informação da Atenção à Saúde Indígena, mantido pela Fundação Nacional de Saúde (FUNASA).

Para os registros que apresentaram diagnóstico secundário, foram priorizadas as pneumopatias, as desidratações e as desnutrições como causa principal. Para as crianças internadas no mesmo hospital, com o mesmo diagnóstico e em dias seguidos (até 15 dias), ou seja, perfazendo mais de um evento de internação, somaram-se os tempos de duração das internações anotados nos vários registros. Estes, para fins da análise, foram transformados em um único caso de internação.

A classificação dos diagnósticos (categoria de três dígitos) foi realizada independentemente por dois investigadores (Orellana e Basta), (Kappa 0,87, IC95\%: 0,79-0,95), utilizando-se a Classificação Estatística Internacional de Doenças e Problemas Relacionados à Saúde, $10^{\mathrm{a}}$ revisão (CID-10). ${ }^{11} \mathrm{~A}$ análise dos dados foi conduzida no programa SPSS, 
Principais causas de internação hospitalar em crianças Suruí menores de 10 anos de idade, por faixa etária, segundo a classificação por capitulo da Classificação Internacional de Doenças, 10. ${ }^{a}$ revisão (CID-10). Cacoal, Rondônia, 2000 a 2004.

\begin{tabular}{|c|c|c|c|c|c|c|c|c|}
\hline \multirow{3}{*}{ Causas de internação hospitalar (CID 10) } & \multicolumn{8}{|c|}{ Faixas etárias (anos) } \\
\hline & \multicolumn{2}{|c|}{$<2$} & \multicolumn{2}{|c|}{$2-4$} & \multicolumn{2}{|c|}{$5-9$} & \multicolumn{2}{|c|}{$0-9$} \\
\hline & $\mathbf{n}$ & $\%$ & $\mathbf{n}$ & $\%$ & $\mathbf{n}$ & $\%$ & $\mathbf{n}$ & $\%$ \\
\hline Doenças do aparelho respiratório (Cap X) & 131 & 53,0 & 61 & 67,8 & 29 & 67,4 & 221 & 58,2 \\
\hline Algumas doenças infecciosas e parasitárias (Cap I) & 97 & 39,3 & 23 & 25,6 & 13 & 30,2 & 133 & 35,0 \\
\hline Algumas afecções originadas no período perinatal (Cap XVI) & 12 & 4,9 & & & & - & 12 & 3,2 \\
\hline Doenças endócrinas, nutricionais e metabólicas (Cap IV) & 4 & 1,6 & 1 & 1,1 & & - & 5 & 1,3 \\
\hline Doenças da pele e do tecido subcutâneo (Cap XII) & 1 & 0,4 & 1 & 1,1 & & - & 2 & 0,5 \\
\hline \multicolumn{9}{|l|}{ Malformações congênitas, deformações e anomalidades } \\
\hline cromossômicas (Cap XVII) & & - & 2 & 2,2 & & - & 2 & 0,5 \\
\hline Doenças do aparelho circulatório (Cap IX) & & - & 1 & 1,1 & & - & 1 & 0,3 \\
\hline Doenças do aparelho digestivo (Cap XI) & 1 & 0,4 & & - & & - & 1 & 0,3 \\
\hline \multicolumn{9}{|l|}{ Lesões, envenenamento e algumas outras } \\
\hline conseqüências de causas externas (Cap XIX) & & . & 1 & 1,1 & & - & 1 & 0,3 \\
\hline \multicolumn{9}{|l|}{ Sintomas, sinais e achados anormais de exames clínicos e de } \\
\hline laboratório, não classificados em outra parte (Cap XVIII) & 1 & 0,4 & & - & & - & 1 & 0,3 \\
\hline Causas externas de morbidade e de mortalidade (Cap XX) & & & & - & 1 & 2,3 & 1 & 0,3 \\
\hline Total & 247 & 100,0 & 90 & 100,0 & 43 & 100,0 & 380 & 100,0 \\
\hline
\end{tabular}

versão 9.0 (SPSS Inc., Chicago IL, USA). Foram realizados testes $t$ de Student, qui-quadrado e Anova. Consideraram-se estatisticamente significativos os $p$-valores $<0,05$.

Esta investigação insere-se em uma pesquisa mais ampla sobre as condições de saúde dos Suruí, com ênfase na epidemiologia da tuberculose.9,12 Foi aprovada pelo Comitê de Ética em Pesquisa da Escola Nacional de Saúde Pública da Fundação Oswaldo Cruz e pela Comissão Nacional de Ética em Pesquisa (CONEP) (Parecer No. 714/2003).

\section{Resultados}

Foram identificados 380 registros de internações de crianças Suruí nos dois hospitais $(61,1 \%$ no público e 38,9\% no privado). O sub-registro de diagnósticos secundários nas AIH foi elevado (98\%). Crianças internadas no mesmo hospital, com o mesmo diagnóstico e em dias subseqüentes, representaram apenas $0,6 \%$ dos registros.

O tempo médio de internação no hospital público (5,8 dias) foi superior àquele do hospital privado ( 3,7 dias) ( $\mathrm{t}=-7,04 ; p<0,001)$. Não foram observadas diferenças significativas no tempo médio de internação entre os sexos $(p=0,30)$.
As internações de crianças do sexo masculino foram mais freqüentes $(56,3 \%)\left(\chi^{2}=6,06 ; p=0,01\right)$. $\mathrm{Na}$ análise das médias de idade entre os sexos, não foram observadas diferenças significativas $(\mathrm{t}=-0,92$; $p=0,36$ ).

As principais causas de internação foram devido a doenças do aparelho respiratório (CID 10, Capítulo X), às infecciosas e parasitárias (CID 10, Capítulo I) e às afecções originadas no período perinatal (CID 10, Capítulo XVI), com $221(58,2 \%), 133(35,0 \%)$ e $12(3,2 \%)$ registros, respectivamente. As demais causas de internação contribuíram, individualmente, com menos de $1,5 \%$ do total cada (Tabela 1 ).

Dentre as doenças do aparelho respiratório, predominaram as broncopneumonias $(40,3 \%)$, seguidas pelas pneumonias $(23,5 \%)$ e pela asma $(15,4 \%)$. No capítulo das doenças infecciosas e parasitárias, prevaleceram as diarréias e gastrenterites $(91,7 \%)$. A tuberculose correspondeu a $2,3 \%$ das internações neste grupo.

Ao longo dos cinco anos analisados, as taxas de internação por doenças do aparelho respiratório variaram entre $10,6-11,9$ por 100 habitantes na faixa etária, com exceção de 2001, quando se registrou um pico de 27,7. As taxas de internações por doenças infecciosas e parasitárias mantiveram-se relativamente constantes, variando entre 7,3-10,9 (Tabela 2). 
Há uma forte concentração de internações em crianças menores de dois anos, o que alcança $65 \%$ do total (Tabela 3). Nos diversos grupos etários, somente para as crianças entre cinco e nove anos foram observadas diferenças nas freqüências de internação segundo sexo $\left(\chi^{2}=5,23 ; p=0,02\right)$. Independente do grupo etário, as doenças do apa- relho respiratório e as doenças infecciosas e parasitárias constituíram, respectivamente, a primeira e a segunda causa principal de internação (Tabela 1). Em relação às doenças do aparelho respiratório, segundo faixa etária, foram observadas as seguintes porcentagens: a) menores de dois anos: broncopneumonias $(46,6 \%)$, pneumonias $(22,1 \%)$ e asma

\section{Tabela 2}

Taxa de internação hospitalar em crianças indígenas Suruí menores de 10 anos de idade, por 100 habitantes na mesma faixa etária, segundo a classificação por capitulo da Classificação Internacional de Doenças, 10ª revisão (CID-10). Cacoal, Rondônia, 2000 a 2004.

\begin{tabular}{|c|c|c|c|c|c|}
\hline \multirow{2}{*}{ Grupo de Causas (CID - 10) } & \multicolumn{5}{|c|}{ Ano } \\
\hline & 2000 & 2001 & 2002 & 2003 & 2004 \\
\hline Doenças do aparelho respiratório (Cap. X) & 10,6 & 27,7 & 11,2 & 11,9 & 11,8 \\
\hline Doenças infecciosas e parasitárias (Cap. I) & 7,3 & 8,6 & 7,9 & 10,9 & 8,6 \\
\hline Todas as causas & 19,7 & 38,4 & 20,4 & 24,8 & 22,2 \\
\hline
\end{tabular}

Tabela 3

Distribuição das crianças Suruí menores de 10 anos de idade internadas, por faixa etária e sexo. Cacoal, Rondônia 2000 a 2004.

\begin{tabular}{|c|c|c|c|c|}
\hline \multirow{2}{*}{$\begin{array}{l}\text { Faixa etária } \\
\text { (anos) }\end{array}$} & \multicolumn{2}{|c|}{ Sexo } & \multirow[t]{2}{*}{ Total } & \multirow[t]{2}{*}{ Qui-quadrado e valor de $p$} \\
\hline & Feminino & Masculino & & \\
\hline \multicolumn{5}{|l|}{$<2$} \\
\hline n (\%) & $114(46,2 \%)$ & $133(53,8 \%)$ & $247(100,0 \%)$ & \\
\hline$\%$ do total & $68,7 \%$ & $62,1 \%$ & $65,0 \%$ & \\
\hline \multicolumn{5}{|l|}{$2-4$} \\
\hline n (\%) & $38(42,2 \%)$ & $52(57,8 \%)$ & $90(100,0 \%)$ & $\chi_{g 15}^{2}=5,23(p=0,02)$ \\
\hline$\%$ do total & $22,9 \%$ & $24,3 \%$ & $23,7 \%$ & \\
\hline \multicolumn{5}{|l|}{$5-9$} \\
\hline n (\%) & $14(32,6 \%)$ & $29(67,4 \%)$ & $43(100,0 \%)$ & \\
\hline$\%$ do total & $8,4 \%$ & $13,6 \%$ & $11,3 \%$ & \\
\hline \multicolumn{5}{|l|}{$0-9$} \\
\hline n (\%) & $166(43,7 \%)$ & $214(56,3 \%)$ & $380(100,0 \%)$ & $\chi^{2}=6,06(p=0,01)$ \\
\hline$\%$ & $100,0 \%$ & $100,0 \%$ & $100,0 \%$ & \\
\hline
\end{tabular}


$(13,7 \%)$; b) dois a quatro anos: pneumonias $(31,1 \%)$, broncopneumonias $(26,2 \%)$ e bronquites $(24,6 \%)$; e c) cinco a nove anos: broncopneumonias $(41,4 \%)$, asma $(31,0 \%)$ e pneumonias $(13,8 \%)$. Quanto às doenças infecciosas e parasitárias, nos três grupos etários houve o predomínio das diarréias e gastrenterites, com porcentagens que oscilaram entre $84,6 \%$ (5 - 9 anos) a 93,8\% (menores de dois anos).

Em relação ao tempo médio de internação entre as faixas etárias analisadas, segundo os sexos combinados, não foram verificadas diferenças significativas $(\mathrm{F}=1,072 ; p=0,343)$.

\section{Discussão}

A despeito de suas reconhecidas limitações, em particular pelo seu caráter seletivo, os registros de internação hospitalar constituem importantes fontes de dados acerca das condições de saúde, da utilização e da qualidade dos serviços, podendo gerar análises relevantes para o aperfeiçoamento de políticas de saúde e estratégias médico-assistenciais. ${ }^{13,14}$ Devido à escassez de informações sobre saúde indígena, análises sobre morbidade hospitalar têm o potencial de trazer subsídios adicionais para o melhor conhecimento das condições e das demandas de saúde dessas populações. ${ }^{6-8,15}$

Conforme destacado em poucos trabalhos que analisaram o padrão de morbidade hospitalar indígena no país, $7,8,15$ os registros de indígenas atendidos na rede SUS, ao serem incorporados às bases nacionais, como no Sistema de Informações Hospitalares (SIH) do SUS, não incluem informação sobre filiação étnica dos pacientes. Em vista disso, para se acessar os dados, é preciso visitar cada um dos hospitais que integram as redes locais que provê atendimento a determinado povo, obtendo-se os dados diretamente nos prontuários ou em outros registros disponíveis. Ainda que se disponha de um sistema de informação sobre a saúde indígena (SIASI), o mesmo apresenta uma série de limitações que impedem o seu uso para esse tipo de análise.2,5

Este estudo evidencia que as doenças do aparelho respiratório, as doenças infecciosas e parasitárias, com destaque para as diarréias e gastrenterites, constituem as principais causas de internação nas crianças Suruí. Além disso, acometem em maior proporção as crianças menores de um ano, reiterando a vulnerabilidade dessa faixa etária. Esse padrão faz-se presente em segmentos de populações que vivem sob precárias condições socioeconômicas e sanitárias, 16-19 o que é, por certo, o caso da população Suruí. Estudos realizados ao longo das últimas duas décadas entre os Suruí têm destacado sérias deficiências nas condições de saneamento e habitação, além de dificuldades de sustentabilidade alimentar.9,10,20 Em 2004, o coeficiente de mortalidade infantil foi de 70 por mil.21 Um inquérito nutricional realizado no âmbito desse projeto evidenciou, em 2005, prevalência de desnutrição de 31,4\% em crianças menores de cinco anos, avaliada por meio do índice estatura para idade (segundo as curvas do National Center for Health Statistics (NCHS) e de $84,0 \%$ de anemia. 10

Desde 1999, a gestão e a execução da atenção à saúde indígena no Brasil estão sob responsabilidade da Fundação Nacional de Saúde (FUNASA), por meio de 34 Distritos Sanitários Especiais Indígenas (DSEI). A atenção é baseada em agentes indígenas de saúde, que atuam nos postos de saúde das aldeias, e equipes multiprofissionais, sediadas nas sedes dos pólos-base, que realizam visitas periódicas às aldeias. Avaliações recentes indicam sérias dificuldades na implementação do modelo.2,22,23 Apesar dos investimentos, a cobertura e a qualidade dos serviços prestados aos indígenas em várias regiões do país apresentam sérias limitações (alta rotatividade de profissionais, dificuldades operacionais e logísticas, etc), com conseqüente comprometimento da atenção básica nas aldeias.

No caso das crianças Suruí, os resultados deste trabalho indicam que ao longo dos anos os indicadores de internação hospitalar se mantiveram praticamente inalterados para as doenças do aparelho respiratório e chegaram mesmo a apresentar um discreto aumento para as doenças infecciosas e parasitárias, em especial as diarréias. Uma vez que esses agravos são considerados sensíveis à atenção básica, constata-se um quadro marcado pela baixa resolubilidade de ações em saúde disponibilizadas nas aldeias Suruí. A elevada porcentagem de internações atribuídas à diarréia constitui exemplo dessa situação, pois, se corretamente administrada no nível de atenção básica, por meio da terapia de reidratação oral, esperar-se-ia redução das internações por essa causa, a exemplo do que se verificou em outras regiões do país e do mundo. ${ }^{24}$ Acrescente-se que, apesar das ações de saneamento estarem contempladas nas metas da atual política de saúde indígena,2,3 prevalecem condições ambientais bastante deficientes nas aldeias Suruí, o que contribui para o quadro desfavorável da saúde da criança na comunidade.

O tempo médio de permanência hospitalar das crianças Suruí é aproximadamente $65 \%$ maior no hospital público em relação ao privado. Esse achado não difere daqueles de outros estudos realizados no 
país.13,25,26 A variação do tempo médio de permanência hospitalar, conforme assinalam Travassos et al.,27 é uma variável de difícil interpretação, podendo estar relacionada a diferenciais de gravidade do quadro clínico dos indivíduos internados em cada hospital, a melhor adequação e uso de tecnologias médicas e de cuidados aos internos, assim como a erros aleatórios.

Apesar de a desnutrição ter sido observada em uma porcentagem elevada de crianças Suruí menores de cinco anos, ${ }^{10}$ e a associação entre infecções respiratórias agudas e gastrenterites e desnutrição grave ser bem conhecida, chama atenção o registro de apenas um caso de desnutrição (possivelmente aguda) na base de dados analisada. Essa ausência sugere a ocorrência de subnotificação de distúrbios nutricionais por parte dos serviços de saúde, o que se manifesta por meio de sua baixa ocorrência como diagnósticos primários ou secundários nas $\mathrm{AIH}$.

Este estudo sugere que, em larga medida, dá-se no caso Suruí a reprodução do modelo médico-assistencial centrado no hospital, amplamente presente no país. Tal modelo se contrapõe às diretrizes da política nacional de atenção básica em saúde, que tem por base uma proposta de atenção integral à saúde, norteada por princípios que visam não só a cura de doenças, mas também a prevenção e a promoção da saúde.2,3 Considerando que, freqüentemente, cerca de um 1/3 do contingente populacional indígena é composto por crianças menores de 10 anos, o foco dos programas e serviços destinados ao atendimento dessas populações deve priorizar intervenções visando a melhoria das condições ambien-

\section{Referências}

1. Pagliaro H, Azevedo MM, Santos RV, organizadores. Demografia dos povos indígenas no Brasil. Rio de Janeiro: Fiocruz; 2005

2. Garnelo L, Macedo G, Brandão LC. Os povos indígenas e a construção das políticas de saúde no Brasil. Brasília, DF: Organização Pan-Americana da Saúde; 2003.

3. Santos RV, Coimbra Jr. CEA. Cenários e tendências da saúde e da epidemiologia dos povos indígenas no Brasil. In: Coimbra Jr. CEA, Santos RV, Escobar AL, organizadores. Epidemiologia e saúde dos povos indígenas no Brasil. Rio de Janeiro: Fiocruz; 2003. p. 13-47.

4. IBGE (Instituto Brasileiro de Geografia e Estatística). Tendências demográficas: uma análise dos indígenas com base nos resultados da amostra dos censos demográficos 1991 e 2000. Rio de Janeiro; 2005. tais, assim como a consolidação da atenção básica nas aldeias, de modo a promover melhoras nos indicadores de saúde dessa faixa etária, levando a uma redução nas taxas de hospitalização por doenças preveníveis.

\section{Agradecimentos}

À equipe de enfermagem do Pólo Base de Cacoal, Distrito Sanitário Especial Indígena Vilhena, pela colaboração. Também à direção do Hospital Materno-Infantil e do Hospital e Maternidade Menino Jesus, pelo acesso aos dados. Ao Conselho Nacional de Desenvolvimento Científico e Tecnológico (CNPq) pelo suporte financeiro (processos nos. 506392/2004-0 e 470850/2004-3) e à Fundação Ford.

5. Sousa MC, Scatena JHG, Santos RV. O Sistema de Informação da Atenção à Saúde Indígena - SIASI: criação, estrutura e funcionamento. Cad Saúde Pública. 2007; 23: 853-62.

6. Coimbra Jr. CEA, Flowers NM, Salzano FM, Santos RV. The Xavante in transition: health, ecology, and bioanthropology in Central Brazil. Ann Arbor: University of Michigan Press; 2002.

7. Escobar AL, Rodrigues AF, Alves CLM, Orellana JDY, Santos RV, Coimbra Jr. CEA. Causas de internação hospitalar indígena em Rondônia. O distrito sanitário especial indígena de Porto Velho (1998-2001). In: Coimbra Jr. CEA, Santos RV, Escobar AL, organizadores. Epidemiologia e saúde dos povos indígenas no Brasil. Rio de Janeiro: Fiocruz; 2003. p. 127-47. 
8. Lunardi RR. Morbidade hospitalar de indígenas Xavante no Distrito Sanitário Especial Indígena Xavante de Mato Grosso (1998-2002) [dissertação mestrado]. Rio de Janeiro: Escola Nacional de Saúde Pública da Fundação Oswaldo Cruz; 2004.

9. Basta PC, Coimbra Jr. CEA, Escobar AL, Santos RV, Alves LC, Fonseca L. Survey for tuberculosis in an indigenous population of Amazonia: the Surui of Rondonia, Brazil. Trans R Soc Trop Med Hyg. 2006; 100: 579-85.

10. Orellana JDY, Coimbra Jr. CEA, Lourenço AEL, Santos RV. Estado nutricional e anemia em crianças Suruí, Amazônia, Brasil. J Pediatr. (Rio J) 2006; 85: 383-8.

11. OMS (Organização Mundial da Saúde). Classificação Estatística Internacional de Doenças e Problemas Relacionados à Saúde - CID: 10. revisão. Tradução : Centro Colaborador da OMS para a Classificação de Doenças em Português. 8. ed. São Paulo: Universidade de São Paulo; 2000.

12. Basta PC. A tuberculose entre o povo indígena Suruí de Rondônia, Amazônia, Brasil [tese doutorado]. Rio de Janeiro: Escola Nacional de Saúde Pública Sérgio Arouca da Fundação Oswaldo Cruz; 2005.

13. Gouvêa CSD, Travassos C, Fernandes C. Produção de serviços e qualidade da assistência hospitalar no Estado do Rio de Janeiro, Brasil - 1992 a 1995. Rev Saúde Pública. 1997; 31: 601-17.

14. Bittencourt AS, Camacho LAB, Leal MC. O sistema de informação hospitalar e sua aplicação na saúde coletiva. Cad Saúde Pública. 2006; 22: 19-30.

15. Diehl E. Agravos na saúde Kaingáng (Terra Indígena Xapecó, Santa Catarina) e a estrutura dos serviços de atenção biomédica. Cad Saúde Pública. 2001; 17: 439-45.

16. Victora CG, Barros FC, Kirkwood BR, Vaughan JP. Pneumonia, diarrhoea and growth in the first four years of life: a longitudinal study of 5.914 Brazilian infants. Am J Clin Nutr. 1990; 52: 391-6.

17. Silva AAM, Gomes UA, Silva RA. Fatores de risco para a hospitalização de crianças de um a quatro anos em São Luís, Maranhão, Brasil. Cad Saúde Pública. 1999; 15: 74957.

Recebido em 28 de junho de 2006

Versão final apresentada em 14 de maio de 2007

Aprovado em 18 de maio de 2007
18. Caetano JRM, Bordinb IAS, Puccinic RF, Peres CA. Fatores associados à internação hospitalar de crianças menores de cinco anos, São Paulo, SP. Rev Saúde Pública. 2002; 36: 285-91.

19. Vanderlei LCM, Silva GAP, Braga JU. Fatores de risco para internamento por diarréia aguda em menores de dois anos: estudo de caso e controle. Cad Saúde Pública. 2003; 19: 455-63.

20. Santos RV, Coimbra Jr. CEA. On the (un)natural history of the Tupí-Mondé Indians: bioantropology and change in the Brazilian Amazon. In: Goodman AH, Thomas LL, editors. Building a new biocultural synthesis: political-economic perspectives on human biology. Michigan: University of Michigan Press; 1998. p. 26994

21. Escobar AL, Coimbra Jr. CEA, Arias MM, Santos RV. Demografia e saúde reprodutiva dos indígenas Suruí, Rondônia. Cienc Saúde Coletiva. 2005; 10 (Suppl 1): 635.

22. Chaves MBG, Cardoso AM, Almeida C. Implementação da política de saúde indígena no pólo-base Angra dos Reis, Rio de Janeiro, Brasil: entraves e perspectivas. Cad Saúde Pública. 2006; 22: 295-305.

23. Garnelo L, Brandão LC. Avaliação preliminar do processo de distritalização sanitária indígena no Estado do Amazonas. In: Coimbra Jr. CEA, Santos RV, Escobar AL, organizadores. Epidemiologia e saúde dos povos indígenas no Brasil. Rio de Janeiro: Fiocruz; 2003. p. 235-57.

24. Victora CG, Bryce J, Fontaine O, Monasch R. Reducing deaths from diarrhoea through oral rehydration therapy. Bull World Health Organ. 2000; 78: 1246-55.

25. Mathias TAF, Soboll MLMS. Morbidade hospitalar em município da região Sul no Brasil em 1992. Rev Saúde Pública. 1996; 30: 224-32

26. Rocha JSY, Simões BJG, Guedes GLM. Assistência hospitalar como indicador da desigualdade social. Rev Saúde Pública. 1997; 31: 479-87.

27. Travassos C, Noronha JC, Martins M. Morbidade hospitalar como indicador de qualidade: uma revisão. Cienc Saúde Coletiva. 1999; 4: 367-38 